\title{
Inflação, Desemprego e Choques Cambiais: Uma Revisão da Literatura Sobre a Curva de Phillips no Brasil
}

\author{
Adolfo Sachsida*
}

\author{
Contents: 1. Introdução; 2. Estudos sobre a Curva de Phillips no Brasil; 3. Dificuldades \\ Inerentes na Estimativa da Curva de Phillips; 4. Conclusão. \\ Keywords: Curva de Phillips, Inflação, Desemprego, Taxa de Câmbio. \\ JEL Code: E31, E24.
}

Este artigo faz uma ampla revisão da literatura sobre a curva de Phillips no Brasil. De maneira geral, os resultados descritos na literatura nacional se mostraram muito sensíveis ao período analisado, ao conjunto de proxies adotadas, ao instrumental econométrico empregado, e a frequência e ao número de defasagens permitidas para as variáveis. Tais resultados lançam dúvidas sobre a adequação da curva de Phillips para descrever a dinâmica inflacionária da economia brasileira.

This article summarizes the studies about the Phillips curve in the Brazilian economy. Overall, the results are very sensitive to the time period, to the proxies adopted, to the econometric approach, and to the frequency and lags allowed to the variables. These results cast some doubt about the ability of the Phillips curve to describe the dynamics of inflation in the Brazilian economy.

\section{INTRODUÇÃO}

Apesar de ter sido originalmente proposta há mais de meio século a correlação negativa entre inflação e desemprego, conhecida como curva de Phillips, permanece no centro do debate macroeconômico atual. A importância dessa relação pode ser comprovada pelo fato de que estudos sobre a curva de Phillips serem comuns na maioria dos bancos centrais ao redor do mundo, principalmente àqueles que adotam o regime de metas de inflação (Hargreaves et alii, 2006). Além disso, Annable (2007) afirma que a curva de Phillips está entre as relações mais importantes estudadas pela macroeconomia.

Uma extensa literatura referente à dinâmica inflacionária é baseada na curva de Phillips novo-keynesiana, ou seja, um modelo de fixação de preços com rigidez nominal que implica que a

* Instituto de Pesquisa Econômica Aplicada (IPEA), Rio de Janeiro. O autor agradece o apoio da Bolsa de Produtividade em Pesquisa do CNPq. E-mail: adolfo.sachsida@ipea.gov.br 
inflação pode ser explicada pela evolução esperada dos custos marginais reais (Cogley e Sbordone, 2006). Em termos empíricos existe um amplo debate econométrico sobre a relevância da curva de Phillips. Se por um lado os estudos de Galí e Gertler (1999) e Galí et alii (2001) apresentam resultados estatísticos favoráveis à curva de Phillips, temos também os estudos de Rudd e Whelan (2005), Lindé (2005) e Bardsen et alii (2004) sugerindo que a relevância empírica da curva de Phillips deve ser considerada fraca.

Para a economia brasileira, vários autores têm pesquisado sobre a habilidade da curva de Phillips em descrever a dinâmica inflacionária nacional. Tal como acontece com a literatura internacional tais trabalhos adotam sempre técnicas estatísticas relacionadas a séries temporais, as diferenças se dão principalmente:

a) nas proxies adotadas para representar a inflação e o custo marginal das empresas; e

b) no método econométrico de séries temporais a ser empregado (co-integração, co-integração com quebra, VAR, modelo de mudança de regime, modelo com parâmetros variáveis, inter alia).

O objetivo deste artigo é elaborar uma ampla revisão da literatura sobre a curva de Phillips no Brasil. Dada a importância da relação entre inflação, desemprego e choques cambiais, parece ser importante que os pesquisadores da área tenham um material que coleta e explicita os principais avanços obtidos nesse campo. Sendo assim, esse trabalho se propõe a ser um ponto de partida para estudos que pretendam analisar a adequação da Curva de Phillips para representar a dinâmica inflacionária brasileira.

Além dessa Introdução, a Seção 2 apresenta uma breve resenha da literatura sobre a curva de Phillips no Brasil. A Seção 3 explicita alguns dos diversos problemas metodológicos relacionados à estimativa da curva de Phillps para a economia brasileira. Por fim, a Seção 4 conclui o artigo.

\section{ESTUDOS SOBRE A CURVA DE PHILLIPS NO BRASIL}

A importância tanto teórica quanto empírica tem despertado a atenção dos pesquisadores brasileiros em relação à curva de Phillips. ${ }^{1}$ Vários artigos tentaram estimar não somente a curva de Phillips para o Brasil, mas também, a partir desta, fazer inferências sobre a adequação da política monetária, do sucesso do regime de metas de inflação, sobre o grau de rigidez salarial da economia brasileira, e da taxa de desemprego que não acelera a inflação.

Num trabalho pioneiro para o Brasil Cysne (1985) conclui pela não-existência de um trade-off entre inflação e capacidade ociosa no longo prazo. Além disso, sugere "Que alterações de política salarial introduzidas entre 1979 e 1983, bem como a ocorrência de duas maxidesvalorizações (...) devem ser incluídas como fatores explicativos da mudança de patamar do nível de inflação ocorrida a partir de 1980".

Cavalcanti (1990) incluiu a volatilidade da inflação na regressão, pois esta poderia afetar o lado da oferta na economia. Dessa maneira, estimou a curva de Phillips, com aversão ao risco, para a economia brasileira com dados trimestrais no período 1976(II)-1989(I). Em sua conclusão, ressalta-se que

a) o ativismo fiscal tende a reduzir o produto e aumentar a inflação de equilíbrio; e

b) "se a volatilidade da inflação cresce com a elevação da taxa de inflação, mais provavelmente o sistema será instável e quanto maior a expansão monetária menor será o nível de produto no longo prazo".

\footnotetext{
${ }^{1}$ Uma revisão mais completa e detalhada sobre os artigos internacionais e nacionais que tratam sobre a curva de Phillips pode ser encontrada em Sachsida (2013)
} 
Os dois artigos acima são importantes referências para compararmos os resultados mais recentes com os resultados de períodos anteriores da economia brasileira. Iremos agora rever estudos mais recentes, que já incorporam dados após a estabilização da economia ocorrida no Plano Real.

Fazendo uso de dados trimestrais para o período 1982 a 1998, Portugal et alii (1999) encontram uma estimativa linear do trade-off entre inflação e desemprego que tem significância estatística, e aparentemente é adequada aos dados da economia brasileira. Como proxy para a inflação adotam o Índice Nacional de Preços ao Consumidor (INPC) elaborado pelo IBGE. Para representar a taxa de desemprego utilizam tanto a Pesquisa Mensal de Emprego (PME) do IBGE como também a Pesquisa de Emprego e Desemprego (PED) do DIEESE/SEADE. Na PED utilizam apenas os dados referentes ao estado de São Paulo. Para obter a inflação esperada estimam vários modelos ARIMA e escolhem aquele que gera a melhor predição para a taxa de inflação. Como o objetivo principal dos autores é estimar a NAIRU (taxa de desemprego que não acelera a inflação), a variável dependente em suas estimativas é a inflação atual menos a inflação esperada para o próximo período. Com os dados do IBGE encontram um coeficiente de 0,185 para a variável (inflação $t-1$ - expectativa de inflação $t-1$ ) e de 0,123 para (inflação $t-2$ - expectativa de inflação $t-2$ ), sendo ambas as variáveis estatisticamente insignificantes. Já para o desemprego encontra-se um coeficiente estatisticamente significante da ordem de $-1,745$.

Num artigo estatisticamente sofisticado, Lima (2003) estima a NAIRU para o Brasil e verifica a estabilidade dos coeficientes da Curva de Phillips brasileira. São estimados dois modelos diferentes em espaço de estado: um com uma NAIRU que muda ao longo do tempo, e outro no qual a NAIRU muda de acordo com a especificação de uma cadeia de Markov oculta. Os resultados econométricos sugerem que a inclinação da Curva de Phillips do Brasil é estável, mas a NAIRU brasileira vem se alterando ao longo do tempo. Os modelos foram estimados com dados trimestrais para o período 1982:01 a 2001:04. A proxy adotada para a inflação foi o INPC e para o desemprego utilizou-se o a taxa de desemprego aberto da PME (IBGE). Para lidar com as possíveis quebras estruturais inerentes a esse período da economia brasileira, o autor estima um modelo TVP (mudança dos parâmetros ao longo do tempo e resíduos $\mathrm{ARCH}$ ) e outro MSR (mudança dos parâmetros ao longo do tempo e regimes de Markov-switching). Os resultados econométricos não possibilitam a rejeição da hipótese de estabilidade da Curva de Phillips. Existe uma relação estatisticamente significante entre o desemprego cíclico e a mudança na taxa de inflação. De acordo com o modelo TVP (MSR) um aumento permanente de 0,5\% do desemprego cíclico, depois de 3 (1) trimestres, reduz em 7,5\% (5\%) a taxa mensal anualizada de inflação. Considerando-se a incerteza sobre qual modelo é o mais adequado, adicionado do intervalo de confiança, temos que qualquer resposta da inflação entre $-11 \%$ e $-4 \%$ não pode ser rejeitada.

Numa análise para o periodo 1995:07 a 2002:12 com dados mensais, Minella et alii (2003) encontram que a inflação esperada reage significativamente à meta de inflação. Eles concluem que esta correlação é um indício de que as metas de inflação são importantes determinantes das expectativas inflacionárias. Como proxy para inflação adotam o Índice de Preços ao Consumidor Ampliado (IPCA), para o desemprego utilizam a taxa de desemprego sazonalmente ajustada de 7 dias (IBGE) (também reportam que os resultados são similares para o caso de dados brutos ou de 30 dias). Encontram os seguintes parâmetros para a curva de Phillips: inflação passada varia entre 0,56 e 0,62 (para o caso em que um segundo lag de inflação é incluído seu coeficiente é de -0.09 mas é estatisticamente igual a zero); desemprego passado varia entre -0.08 (não significativo) e $-0,09$ (significativo, ocorre quando se inclui a segunda defasagem da inflação).

Fasolo e Portugal (2004) testam a relação entre inflação e emprego no Brasil com o uso de 4 hipóteses novo-keynesianas:

i) os agentes não possuem racionalidade perfeita;

ii) imperfeição na formação de expectativas pode ser determinante no componente inercial da inflação brasileira;

iii) inflação possui componente inercial autônomo; e 
iv) relações não-lineares entre inflação e desemprego fornecem melhores resultados para a economia nos últimos 12 anos.

Os testes econométricos confirmam as 4 hipóteses. A curva de Phillips é estimada com o uso do filtro de Kalman, e a relação entre inflação e expectativas é verificada por um modelo de mudança de regime. 0 artigo faz uso de dados mensais para o período 1990:01 a 2002:08 e são adotadas as seguintes proxies: IPCA para inflação e desemprego aberto sazonalmente ajustado, 30 dias, do IBGE para a variável custo marginal das empresas. Concluem que a Curva de Phillips para o Brasil deve ser estimada levando em consideração algum tipo de não-linearidade. Encontram os seguintes parâmetros para a curva de Phillips: inflação passada $=0,13$, inflação futura $=0,82$ e hiato do desemprego $=74,23$. Usando o subperíodo jan/90 a jun/94: inflação passada $=0,30$, inflação futura $=0,44$. E para o subperíodo jan/95 a ago/02: inflação passada $=0,10$ e inflação futura $=-0.04$ (não significante).

Muinhos (2004) adota o hiato do produto para representar os custos marginais na curva de Phillips. Utiliza dados trimestrais do período 1994:04 a 2002:02 e usa o IPCA como proxy da inflação, e estima o hiato do produto como sendo o desvio do PIB de sua tendência linear. Para a representar a expectativa de inflação estima um processo ARMA. De maneira interessante, não inclui a inflação passada e a expectativa de inflação juntas nas mesmas regressões. Para uma curva de Phillips linear encontra os seguintes parâmetros: inflação passada $=0,51$, e hiato do produto $=0,28$; em outra regressão encontra também: expectativa de inflação $=1,18$, e hiato do produto $=0,35$. Para uma curva de Phillips não-linear: inflação passada $=0,45$, e hiato do produto $=0,41$; ou ainda: expectativa de inflação $=$ 1,29 , e hiato do produto $=0,31$.

Alves e Areosa (2005) fazem uma contribuição teórica ao incluir a meta de inflação na curva de Phillips. Eles derivam a curva de Phillips novo-keynesiana incorporando a indexação não apenas por meio da inflação passada, mas também pela meta de inflação. Como proxies adotam o IPCA para a inflação, e constroem uma variável que representa o custo marginal agregado das firmas (índice de salário real vezes a força de trabalho ocupada, tudo dividido pela parcela da renda do trabalho vezes o PIB). Essa variável refere-se apenas a região metropolitana de São Paulo (dados da Fundação SEADE). Usam dados trimestrais do período 1995-2004, e encontram os seguintes parâmetros: custo marginal $=-0,11$ (não-significativo a 10\%); meta de inflação = 0,68 (significativo a 1\%).

Schwartzman (2006) realiza estimativas da Curva de Phillips para o Brasil a partir de dados de preços desagregados fazendo uso do método de mínimos quadrados em três estágios. Foram usados dados trimestrais para diferentes amostras começando em 1997, 1998 e 1999, e terminando todas elas no terceiro trimestre de 2003. Como proxy para o custo marginal das empresas adota a utilização da capacidade da indústria (FGV), para a inflação presente e passada utiliza o IPCA cheio. Já as expectativas de inflação foram modeladas a partir de um VAR. A grande novidade deste estudo reside no fato de regredir conjuntamente três grupos de preços (comercializáveis, não-comercializáveis e monitorados) na estimação da curva de Phillips. Foram estimadas variações do modelo básico para testar o efeito de algumas das diferentes opções de modelagem realizadas pela literatura. De forma geral, não foi possível rejeitar a hipótese de verticalidade de longo prazo da curva de Phillips, isto é, esta parece ser uma boa hipótese de trabalho ao se analisar a economia brasileira. Os parâmetros encontrados foram os seguintes para a equação de não-comercializáveis: capacidade instalada $=0,71$ a 1,27 , e inflação passada $=0,39$ a 0,50. E para a equação de comercializáveis: inflação passada $=0,37$ a 0,56 .

O estudo de Tombini e Alves (2006) desagrega o IPCA em dois componentes: mercado livre e preços monitorados. Usando um filtro de Kalman estimam uma curva de Phillips híbrida com parâmetros variáveis. Encontram que vários coeficientes se movem para patamares distintos em pelo menos duas ocasiões: na mudança para câmbio flexível em 1999 e no meio de 2002. Logaritmizam todas as variáveis para o período Janeiro de 1996 a Janeiro de 2006, como proxy do custo marginal adotam o hiato do produto. Encontram os seguintes parâmetros: inflação passada: 0,3 a 0,1; e inflacao futura: idem.

$\mathrm{O}$ artigo de Mendonça e dos Santos (2006) avalia se a incorporação de uma medida de credibilidade da política monetária melhora o poder de previsão da Curva de Phillips brasileira no período posterior 
à introdução do regime de metas para inflação. Os resultados encontrados indicam que a utilização de uma medida de credibilidade pode prover um modelo com qualidade de previsão superior àquelas obtidas por modelos que impõem uma relação estável entre a inflação e as expectativas de inflação. Como proxy para o desemprego adotam a taxa de desemprego aberto da PED (DIEESE/SEADE) da região metropolitana de São Paulo. A expectativa de inflação é estimada utilizando a série de expectativas de inflação (medidas pelo IPCA) disponibilizadas pelo Banco Central do Brasil. Os parâmetros encontrados são: entre 0,43 e 0,96 para a inflação esperada; entre $-0,09$ e -0,16 para o hiato do desemprego (taxa natural - taxa de desemprego); e entre -0,01 e -0,08 para a primeira diferença do hiato do desemprego.

Areosa e Medeiros (2007) derivam e estimam um modelo estrutural para inflação numa economia aberta. O modelo representa a curva de Phillips novo-keynesiana padrão e uma curva híbrida. Na parte econométrica usam dados mensais para o período 1995:01 a 2003:09, e adotam duas proxies para o custo marginal das empresas: a renda do trabalho na produção, e o hiato do produto (produção industrial do IBGE regredida contra 11 dummies sazonais e uma tendência linear), para a inflação adota-se o IPCA. Resultados econométricos para a Economia Fechada (estimativa por GMM):

(i) a inflação passada é um componente não negligível, com estimativas consistentes ao redor de 0,45;

(ii) a inflação futura é dominante, com valores ao redor de 0,53; e

(iii) o impacto dos custos marginais não é estatisticamente significante, e tem um efeito negligível.

Já para a Economia Aberta os resultados indicam que:

i) a inflação passada tem coeficiente menor do que no caso da economia fechada, variando entre 0,10 e 0,37 ;

ii) a inflação futura mostra valores maiores que no caso da economia fechada, variando entre 0,63 e 0,81 ; e

iii) o impacto dos custos marginais, apesar de serem em magnitude irrelevante, é positivo e estatisticamente significante.

Arruda et alii (2008) adotam modelos não-lineares da Curva de Phillips para fins de previsão. Eles argumentam que a curva de Phillips ampliada com efeito limiar (threshold) é a que apresenta o melhor desempenho em termos preditivos. Segundo os autores, num regime com taxa de inflação de quatro meses abaixo de $0,17 \%$, o efeito da inércia inflacionária e do repasse cambial são estatisticamente insignificantes. Contudo, no regime em que a inflação dos quatro meses passados supera a marca de $0,17 \%$ os efeitos do repasse cambial, e da inércia inflacionária, aumentam e se tornam estatisticamente significantes. Mas, em ambos os casos, o hiato do produto não se mostrou estatisticamente significante.

Mazali e Divino (2009) estimam a curva de Phillips para a economia brasileira, com dados trimestrais, para o período 1995:01 a 2008:04. Todas as variáveis são logaritmizadas, e adotam as seguintes proxies: IPCA para a inflação, e a taxa de desemprego aberta, sazonalmente ajustada, para a região metropolitana de São Paulo (DIEESE) para o custo marginal das empresas. Já a inflação esperada é estimada por variáveis instrumentais. Estimam os parâmetros da curva de Phillips por GMM com desvios-padrões robustos. Este artigo é um dos poucos a se preocupar seriamente com a ordem de integração das séries, concluindo após uma bateria de testes que todas as variáveis são estacionárias. Os resultados econométricos são consistentes com a teoria, mostrando um bom grau de ajustamento da curva de Phillips aos dados brasileiros. Os parâmetros encontrados foram de 0,59 para a inflação passada, de 0,44 para a inflação futura, e de -0,13 para o desemprego.

Sachsida et alii (2009) estimam a curva de Phillips por meio de modelos não-lineares, mostrando que os resultados são extremamente sensíveis tanto as proxies adotadas para representar o custo marginal das empresas, como também as especificações de linearidade adotadas. Corroborando esse resultado 
Sachsida et alii (2009), num estudo de dados de painel para seis regiões metropolitanas brasileiras, apontam para a inadequação da curva de Phillips em representar a dinâmica inflacionária brasileira.

Areosa et alii (2011) salientam a importância de modelos não-lineares para explicar a evolução da dinâmica inflacionária brasileira. Eles concluem pela existência de dois regimes: o primeiro de baixa incerteza e o segundo de alta incerteza, sendo que a inércia inflacionária só seria relevante no regime de baixa incerteza (desaparecendo no regime de alta incerteza). Já o hiato do produto só seria significante quando a incerteza inflacionária fosse alta. Isto é, a variável de custo marginal (hiato do produto nesse caso) teria habilidade para explicar a dinâmica inflacionária apenas em regimes de alta incerteza.

Adotando uma modelagem VAR da curva de Phillips com choques cambiais, no período março de 2003 a março de 2011, Schettini et alii (2012) encontram que:

a) 0 impacto de um choque cambial na inflação (pass-through cambial) é de aproximadamente 0,04 pontos percentuais (p.p.) na inflação do mês seguinte ao choque (ou 0,48 p.p. na inflação anualizada);

b) Um choque médio na taxa de desemprego demora ao redor de 18 meses para desaparecer;

c) Uma inovação de 0,058 p.p na expectativa de inflação é carregada para a inflação, que atinge um máximo de 0,049 p.p. no mês seguinte ao choque (o que corresponde a um acréscimo na inflação anualizada de 0,58 p.p.); e

d) Choques na série de inflação não afetam a taxa de desemprego. Isto é, mais inflação não reduz a taxa de desemprego.

Mendonça et alii (2012) estimam a curva de Phillips novo-Keynesiana (NKPC) para o Brasil pelo método GMM-HAC. Para garantir a robustez dos resultados vários procedimentos foram realizados. De maneira geral, apenas um resultado parece ser robusto o bastante: a expectativa futura de inflação e a inflação passada têm relevância na dinâmica do processo inflacionário. Contudo, o papel das expectativas parece aumentar no período mais recente a partir de 2002. Quando a amostra se estende com dados a partir de 1995, o efeito das expectativas é menor ou semelhante ao da inércia inflacionária. Além disso, para a maior parte das regressões estimadas, não foi possível rejeitar a hipótese derivada da forma estrutural da NKCP de que a soma dos coeficientes da inflação passada e da expectativa de inflação seja igual a unidade. Contudo, no conjunto, os resultados parecem sugerir que o processo inflacionário brasileiro não guarda relação próxima com a NKPC.

\section{DIFICULDADES INERENTES NA ESTIMATIVA DA CURVA DE PHILLIPS}

De maneira geral, podemos estimar a curva de Phillips de acordo com a equação 1.

$$
\pi_{t}=\beta_{1} \pi_{t-1}+\beta_{2} E_{t} \pi_{t+1}+\beta_{3} x_{t}+\beta_{4} z_{t}+\varepsilon_{t}
$$

onde $\pi_{t}$ é a taxa de inflação no período $t, E_{t} \pi_{t+1}=E\left[\pi_{t+1} \mid I_{t}\right.$ é a esperança matemática da taxa de inflação para o próximo período, formada com base no conjunto de informação $I_{t}$, $x_{t}$ é alguma variável que representa o custo marginal da empresa, $z_{t}$ é uma variável que representa um choque de oferta (geralmente um choque cambial), e $\varepsilon_{t}$ é o erro que assume-se ser independente e identicamente distribuído.

A forma reduzida representada pela equação (1) está associada a forma estrutural derivada em Blanchard e Galí (2007):

$$
\pi_{t}=\frac{1}{1+\beta} \pi_{t-1}+\frac{\beta}{1+\beta} E_{t} \pi_{t+1}-\frac{\lambda(1-\alpha)(1-\gamma) \phi}{\gamma(1+\beta)} x_{t}+\frac{\alpha \lambda}{1+\beta} z_{t}+\varepsilon_{t}
$$

onde $\beta \in(0,1)$ é o fator de desconto intertemporal, $\alpha \in(0,1)$ assinala que a função de produção da economia apresenta retorno constante de escala, $\lambda \equiv \theta^{-1}(1-\theta)(1-\beta \theta)$ onde $\theta$ é fração das firmas 
que não reajustam o preço em cada período, $\gamma$ é o coeficiente que mede a rigidez salarial e $\phi$ indica a declividade da curva de oferta de trabalho. Tendo em vista a abordagem econométrica utilizada por Blanchard e Galí (2007), bem como aquelas que serão empregadas neste estudo para estimar a equação (1), o único coeficiente estrutural que pode ser identificado é o fator de desconto $\beta$, o restante não pode ser recuperado. Baseado no fato de que $\beta \in(0,1)$, pode-se notar que a forma estrutural expressa por (1') impõe restrições sobre os parâmetros $\beta_{1}$ e $\beta_{2}$ da forma reduzida de modo que:

$$
\beta_{1}, \beta_{2}, \in(0,1) \text { e } \beta_{1}+\beta_{2}=1
$$

Apesar de possuir uma representação simples, a curva de Phillips apresenta razoáveis dificuldades para sua estimação e implementação. A primeira dificuldade refere-se a qual conjunto de variáveis devemos adotar como proxies para inflação, expectativa de inflação, custo marginal da empresa, e choque de oferta. Essa escolha não é trivial, e vários estudos escolhem diferentes conjuntos de variáveis, não havendo ainda uma metodologia de escolha que sugira quais variáveis devem ser adotadas. No caso brasileiro, tal dificuldade é maior ainda devido a precariedade de várias séries estatísticas, aliada a grande instabilidade econômica que caracterizou o começo da década de 1990.

Outra dificuldade estatística associada à curva de Phillips refere-se ao método pelo qual devemos estimar a equação (1). Ocorreu mudança de regime no período analisado? Os parâmetros são variáveis no tempo? A variável $x$ é exógena na equação (1) ou devemos estimar um sistema de equações? São dúvidas pertinentes que surgem durante a estimativa da curva de Phillips para a economia brasileira. Além disso, devemos nos lembrar que determinadas variáveis econômicas só afetam as demais depois de decorridos determinados espaços de tempo. Isto é, quantas defasagens devem ser adotadas na estimativa da equação (1)? As perguntas elaboradas nesse parágrafo não encontram respostas definidas na literatura. Sendo assim, cada pesquisador adota seu próprio critério de escolha. Claro que tais critérios são sempre embasados em motivações teóricas e/ou empíricas. Mesmo assim, a ampla gama de resultados encontrados, descrita na seção de revisão de literatura, mostra como tais escolhas podem ter impacto grande nos resultados finais.

Uma das grandes barreiras à estimativa da curva de Phillips refere-se a escolha das proxies para representar as variáveis descritas na equação (1). Em primeiro lugar devemos escolher uma variável que representa a inflação. A inflação medida pelo Índice de Preços ao Consumidor Ampliado (IPCA) é a proxy padrão para os estudos que estimam a curva de Phillips brasileira após a implementação do regime de metas de inflação. O problema mais sério com o uso dessa proxy é que a taxa de inflação medida pelo IPCA não é calculada para o Brasil como um todo, mas apenas para 11 regiões metropolitanas $3 .^{2}$ Ou seja, assume-se que a dinâmica da evolução dos preços nos mais de 5.000 municípios brasileiros possam ser representados por essas 11 regiões metropolitanas. Sachsida et alii (2009) tentaram minimizar esse problema, estimando 3 diferentes curvas de Phillips: uma para o estado do Rio de Janeiro, uma para o estado de São Paulo, e outra para o Brasil. Na curva de Phillips para o Rio de Janeiro adotou-se a inflação carioca em conjunto com outras variáveis estaduais, sendo o mesmo procedimento feito para o estado de São Paulo. Sachsida e Mendonça (2009) também tentaram endereçar o mesmo problema, e estimaram uma curva de Phillips com dados de painel para 6 localidades onde haviam estimativas de taxas de inflação e de desemprego locais. Contudo, ainda não existem razões teóricas que estabeleçam o IPCA como a correta medida de inflação na curva de Phillips. ${ }^{3}$

Em segundo lugar, não é fácil escolher uma variável que represente a expectativa de inflação. No Brasil, duas estratégias são comumente empregadas:

\footnotetext{
${ }^{2}$ As onze regiões metropolitanas são: Belém, Fortaleza, Recife, Salvador, Belo Horizonte, Rio de Janeiro, São Paulo, Curitiba e Porto Alegre, Brasília e município de Goiânia.

${ }^{3}$ Deve-se ressaltar que na sua formulação original a curva de Phillips referia-se a inflação salarial, e não a uma taxa de inflação da economia.
} 
a) adota-se a mediana das previsões de inflação que estão presentes no relatório FOCUS do Banco Central do Brasil. O problema com essa estratégia é que essa informação somente está disponível a partir de 2001; e

b) estima-se a expectativa de inflação por alguma metodologia econométrica. O problema com essa abordagem é que ela é extremamente $a d$ hoc.

Quais variáveis deveriam entrar nessa regressão? Quantas defasagens de cada uma devem ser inclusas? Ou seja, encontrar uma proxy para a expectativa de inflação não é tarefa fácil. Os resultados presentes em Sachsida et alii (2010) mostram que o uso de diferentes proxies para a expectativa de inflação tem impacto direto nos coeficientes estimados da inflação passada e da expectativa de inflação na curva de Phillips (apesar de que em ambas as formulações não se pode rejeitar que a soma desses coeficientes seja igual a um).

Um terceiro problema surge na representação do custo marginal das empresas. Apesar de Mankiw (2001) criticar o uso da variável hiato do produto para representar o custo marginal das empresas, o fato é que muitos estudos continuam adotando essa proxy. Temos que diferentes artigos adotam diferentes variáveis como proxy para o custo marginal das empresas. Além do hiato do produto, a massa salarial, a utilização da capacidade instalada da indústria, a taxa de desemprego, e o hiato da taxa de desemprego, são variáveis que aparecem com certa frequência para representar o custo marginal das empresas. Desnecessário dizer que estudos que adotam o hiato (seja do produto ou do desemprego) como proxy devem se preocupar com outro problema, qual seja, como calcular esse hiato? Geralmente, tal hiato é construído com o uso de filtros estatísticos. Mas por mais elaborados que tais filtros sejam, eles ainda são ad hoc.

A proxy mais adotada para representar o custo marginal das empresas costuma ser a taxa de desemprego. Contudo, para o caso brasileiro, existem dois grandes problemas com essa série. 0 primeiro problema é que a série de desemprego para o Brasil, calculada pelo IBGE, sofreu alterações metodológicas e a séria antiga de desemprego foi interrompida em 2002, ao mesmo tempo a série nova de desemprego só foi calculada a partir de outubro de 2001. Assim, os estudos que adotam a taxa de desemprego para estimar a curva de Phillips brasileira costumam usar como proxy a taxa de desemprego de São Paulo calculada pela Fundação SEADE em conjunto com o DIEESE. Tal procedimento é uma simplificação adotada por tais estudos, uma vez que supõe que a dinâmica de emprego no Brasil é similar a dinâmica de São Paulo. Isso é certamente uma limitação importante, já que tal taxa de desemprego não necessariamente representa o Brasil como um todo. O segundo problema é que, mesmo a série nova de desemprego para o Brasil é calculada levando em consideração apenas 6 regiões metropolitanas. (Regiões metropolitanas de Recife, Salvador, Belo Horizonte, Rio de Janeiro, São Paulo e Porto Alegre).

Por fim, temos que escolher uma proxy para representar o choque de oferta. Na literatura, o mais comum é adotar o choque cambial como proxy para esta variável. Ainda assim, surgem dúvidas: devemos usar a taxa de câmbio em nível ou sua taxa de crescimento? Quanto tempo leva para um choque cambial afetar a inflação? Evidentemente a resposta a essas perguntas tem implicações diretas nos resultados econométricos encontrados. Outro detalhe: existem indícios de que desvalorizações cambiais não afetam rapidamente o IPCA. Seria o caso de alterar então a proxy para a inflação? Como se nota, apesar de teoricamente simples, a operacionalização e estimação da curva de Phillips apresenta razoáveis dificuldades técnicas.

\section{CONCLUSÃO}

Este trabalho reviu a literatura sobre a curva de Phillips no Brasil. De maneira geral, podemos afirmar que nossa pesquisa revisou os resultados encontrados por diversos autores que tentaram estimar a curva de Phillips novo-Keynesiana para a economia brasileira. Ao longo dos anos diversas 
metodologias econométricas distintas foram empregadas: regressões lineares e não-lineares, modelos autoregressivos, modelos com parâmetros variáveis, dados de painel, séries temporais frequentistas, regressões bayesianas, regressões de transição suave (STR), entre outras técnicas foram adotadas. Não menos prolixa foram as diferentes séries de variáveis usadas como proxy para a estimativa da curva de Phillips.

Como foi observado na seção de revisão de literatura, as diversas estimativas da curva de Phillips para o Brasil chegam a diferentes conclusões. A gama de parâmetros encontrados para representar o efeito do custo marginal das empresas sobre a taxa de inflação é enorme: variando de efeito nenhum (ou positivo) em alguns estudos para um efeito negativo (e estatisticamente significante) em outros estudos. Igualmente amplos são os resultados referentes aos coeficientes estimados da inflação passada e da expectativa de inflação. O efeito do choque cambial sobre a inflação também é controverso. Sendo que tal efeito depende muito do número de defasagens permitidas para o choque cambial. Além disso, pode ocorrer do choque cambial não ser estatisticamente significante (ou quando o for apresentar sinal oposto ao esperado) para explicar a inflação brasileira no curto prazo. Apesar de contra-intuitivo esse resultado não é novo na literatura internacional. Allsopp et alii (2006) já haviam encontrado resultado semelhante para o Reino Unido, e Bailliu e Fujii (2004) argumentam que o grau de pass-through diminui em ambientes de baixa inflação. Além disso, para o Brasil, Nogueira Junior (2010) sugere que uma das consequências das baixas taxas de inflação observadas no Brasil pode ser a redução do pass-through cambial.

O único resultado geral que encontramos refere-se a restrição sugerida por Blanchard e Galí (2007) (de que a soma dos coeficientes da inflação passada e da expectativa de inflação devem ser igual a 1). Todos os estudos que testaram essa hipótese confirmaram a adequação da restrição proposta.

De maneira mais ampla, parece ficar a dúvida da adequação do uso da curva de Phillips para descrever a dinâmica inflacionária brasileira no período recente. Esse resultado não deixa de ser importante, afinal a descrição da inflação como que seguindo a dinâmica proposta pela curva de Phillips é uma suposição padrão na elaboração de política econômica. Talvez seja o momento de tentarmos outras formas de descrever a dinâmica inflacionária da economia brasileira.

\section{BIBLIOGRAPHY}

Allsopp, C., Kara, A., \& Nelson, E. (2006). UK inflation target and exchange rate. Working paper, Federal Reserve Bank of St. Louis. http://research. stlouisfed.org/wp/2006/2006-030.pdf.

Alves, S. A. L. \& Areosa, W. D. (2005). Targets and inflation dynamics. Working Paper Series 100, Central Bank of Brazil.

Annable, J. (2007). Adjusting wages for price inflation: The rational-arrangements Phillips curve. Texto disponível no SSRN: http://ssrn. com/abstract=1045321.

Areosa, W. D., McAleer, M., \& Medeiros, M. (2011). Moment-based estimation of smooth transition regression models with endogenous variables. Journal of Econometrics, 165:100-111.

Areosa, W. D. \& Medeiros, M. (2007). Inflation dynamics in Brazil: The case of a small open economy. Brazilian Review of Econometrics, 27:131-166.

Arruda, E. F., Ferreira, R. T., \& Castelar, I. (2008). Modelos lineares e não lineares da curva de Phillips para previsão da taxa de inflação no Brasil. In ANPEC, editor, Anais do XXXVI Encontro Nacional de Economia .

Bailliu, J. \& Fujii, E. (2004). Exchange rate pass-through and the inflation environment in industrialized countries: An empirical investigation. Working Paper 21, Bank of Canada. 
Bardsen, G., Jansen, E. S., \& Nymoen, R. (2004). Econometric evaluation of the New Keynesian Phillips curve. Oxford Bulletin of Economics and Statistics, 66:671-86.

Blanchard, O. \& Galí, J. (2007). Real wage rigidities and the New Keynesian model. Journal of Money, Credit and Banking, 39:35-65.

Cavalcanti, R. O. (1990). Inflação, estagnação e incerteza: Teoria e experiência brasileira. 14o Prêmio BNDES de Economia (10. Colocado). Rio de Janeiro.

Cogley, T. \& Sbordone, A. M. (2006). Trend inflation and inflation persistence in the New Keynesian Phillips curve. Staff Report 270, Federal Reserve Bank of New York Staff Reports.

Cysne, R. P. (1985). A relação de Phillips no Brasil: 1964-66 × 1980-84. Revista Brasileira de Economia, 39:401-22.

Fasolo, A. M. \& Portugal, M. S. (2004). Imperfect rationality and inflationary inertia: A new estimation of the phillips curve for Brazil. Estudos Econômicos, 34:725-776.

Galí, J. \& Gertler, M. (1999). Inflation dynamics: A structural econometric analysis. Journal of Monetary Economics, 44:195-222.

Galí, J., Gertler, M., \& Lopez-Salido, J. D. (2001). European inflation dynamics. European Economic Review, 45:1237-1270.

Hargreaves, D., Kite, H., \& Hodgetts, B. (2006). Modelling New Zealand inflation in a Phillips curve. Reserve Bank of New Zealand: Bulletin, 69:23-37.

Lima, E. C. R. (2003). The NAIRU, unemployment and the rate of inflation in Brazil. Revista Brasileira de Economia, 57:899-930.

Lindé, J. (2005). Estimating New-Keynesian Phillips curves: A full information maximum likelihood approach. Journal of Monetary Economics, 52:1135-1149.

Mankiw, N. G. (2001). The inexorable and mysterious tradeoff between inflation and unemployment. Economic Journal, 111:C45-C61.

Mazali, A. A. \& Divino, J. A. (2009). Real wage rigidity and the New Phillips curve: The Brazilian case. Working paper, Catholic University of Brasilia.

Mendonça, H. F. \& dos Santos, M. A. L. (2006). Credibilidade da política monetária e a previsão do trade-off entre inflação e desemprego: Uma aplicação para o Brasil. Revista EconomiA, 7:293-306.

Mendonça, M. J. C., Sachsida, A., \& Medrano, L. (2012). Inflação × desemprego: Novas evidências para o Brasil. Economia Aplicada, 16:475-500.

Minella, A., Freitas, P. S., Goldfajn, I., \& Muinhos, M. K. (2003). Inflation targeting in Brazil: Constructing credibility under exchange rate volatility. Journal of International Money and Finance, 22:1015-1040.

Muinhos, M. K. (2004). Inflation targeting in an open financially integrated emerging economy: The case of Brazil. Estudos Econômicos, 34:269-296.

Nogueira Junior, R. P. (2010). Inflation environment and lower exchange rate pass-through in Brazil: Is there a relationship? Revista Brasileira de Economia, 64:49-56.

Portugal, M. S., Madalozzo, R. C., \& Hillbrecht, R. O. (1999). Inflation, unemployment and monetary policy in Brazil. In SBE, editor, Encontro Brasileiro de Econometria. 
Rudd, J. \& Whelan, K. (2005). New tests of the New Keynesian Phillips curve. Journal of Monetary Economics, 52:1167-1181.

Sachsida, A. (2013). Inflação, desemprego e choques cambiais: Uma revisão da literatura sobre a curva de Phillips no Brasil. Texto para discussão, IPEA.

Sachsida, A. \& Mendonça, M. J. C. (2009). Reexaminando a curva de Phillips brasileira com dados de seis regiões metropolitanas. Texto para discussão, IPEA.

Sachsida, A., Mendonça, M. J. C., \& Meddrano, L. (2010). Inflação, desemprego e choques cambiais: Novas evidências para o Brasil. Texto para discussão, IPEA.

Sachsida, A., Ribeiro, M., \& dos Santos, C. H. (2009). A curva de Phillips e a experiência brasileira. Texto para discussão, IPEA.

Schettini, B. P., Gouvea, R. R., \& Sachsida, A. (2012). Inflação, desemprego e choques cambiais: Estimativas VAR para a economia brasileira. Texto para discussão, IPEA.

Schwartzman, F. F. (2006). Estimativa de curva de Phillips para o Brasil com preços desagregados. Economia Aplicada, 10:137-155.

Tombini, A. A. \& Alves, S. A. L. (2006). The recent Brazilian disinflation process and costs. Working Paper Series 109, Central Bank of Brazil. 